\title{
Searches for R-Parity Violating SUSY at ATLAS and CMS
}

\section{David Morse*}

Northeastern University (US)

E-mail: david.michael.morsedcern.ch

\begin{abstract}
The violation of R-parity allows new signatures to be pursued in the search for supersymmetry at the CERN LHC. This article highlights the latest results from the ATLAS and CMS experiments using $20 \mathrm{fb}^{-1}$ of $8 \mathrm{TeV}$ proton-proton LHC collision data of searches for R-parity violating SUSY scenarios with baryon and lepton number violation. The results presented are for dedicated searches for resonances, as well as a systematic analysis of the constraints placed on R-parity violating models with lepton flavour violation, and in terms of production of squarks, gluinos, charginos, neutralinos, and sleptons within R-parity violating SUSY models.
\end{abstract}

The European Physical Society Conference on High Energy Physics 22-29 July 2015

Vienna, Austria

${ }^{*}$ Speaker. 


\section{Introduction}

Supersymmetry (SUSY) represents an extension of the standard model (SM) with a symmetry between fermions and bosons in the form of SUSY super-partners of SM particles. If one writes a minimal extension to the SM which includes SUSY, the super-potential contains four terms which violate lepton or baryon number [1], as seen in Equation 1.1.

$$
W \supset \frac{1}{2} \lambda_{i j k} L_{i} L_{j} \bar{E}_{k}+\lambda_{i j k}^{\prime} L_{i} Q_{j} \bar{D}_{k}+\frac{1}{2} \lambda_{i j k}^{\prime \prime} \bar{U}_{i} \bar{D}_{j} \bar{D}_{k}+\varepsilon_{i} L_{i} H_{2}
$$

The first and last terms (hereafter referred to as LLE and LH, respectively) violate lepton number (LFV), while the second and third terms (hereafter referred to as LQD and UDD, respectively) violate baryon number (BFV).

Unconstrained values of the new Yukawa couplings $\lambda$ and $\varepsilon$ can lead to rapid proton decay, and lepton flavor violating decays, both of which are highly constrained by experimental results. These terms are often avoided in SUSY models by enforcing a new U(1) parity called R-Parity, defined in Equation 1.2. Conservation of R-Parity disallows interactions from these terms of the super-potential.

$$
\begin{aligned}
P_{R}=(-1)^{(2 s+3 B+L)}= & 1 \text { SM Particles } \\
& -1 \text { SUSY Particles }
\end{aligned}
$$

There is, however, no a priori need for R-Parity conservation (RPC), so long as the coupling strength of the new interactions is small enough so as to be compatible with the aforementioned experimental bounds.

R-Parity Violation (RPV) leads to a rich variety of new SUSY signals. In particular, in contrast to RPC SUSY models, the lightest supersymmetric particle (LSP) is not stable, and will decay to SM particles. This leads to signatures with little missing energy in the final state signatures, a classic signature of RPC SUSY. The final states can be very diverse, with 0-4 leptons and 0-10 jets possible. Example diagrams of RPV signatures can be seen in Figure 1.
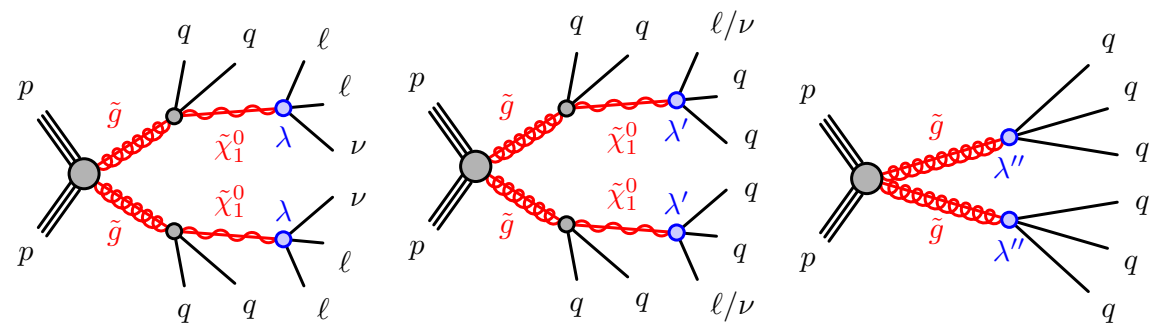

Figure 1: Example diagrams of RPV signatures

This article highlights new results in RPV SUSY searches from the ATLAS and CMS Collaborations. 


\section{Searches}

\subsection{ATLAS Run 1 reinterpretation}

ATLAS has performed a reinterpretation of multiple existing LHC Run 1 searches using simplified models and the phenomenological minimally supersymmetric standard model (pMSSM) for all LFV RPV terms [3].

\subsubsection{LLE gluino}

The first simplified model deals with gluino production with decay via the LLE operator. A representative diagram for this production and decay can be seen in the left-most diagram of Figure 1. The analyses are reinterpreted as a function of the branching ratios of the left-handed (right-handed) super-fields L (E) to tau leptons, covering a large portion of the parameter space of the $\lambda_{i j k}$ operator, as can be seen in the left-most plot of Figure 2. Further, three different values of the ratio of the neutralino to gluino mass $\mathrm{m}\left(\widetilde{\chi}_{1}^{0}\right) / \mathrm{m}(\tilde{g})$ are considered, namely $0.1,0.5$ and 0.9 . The limit plots for 0.1 and 0.9 are also shown in Figure 2.
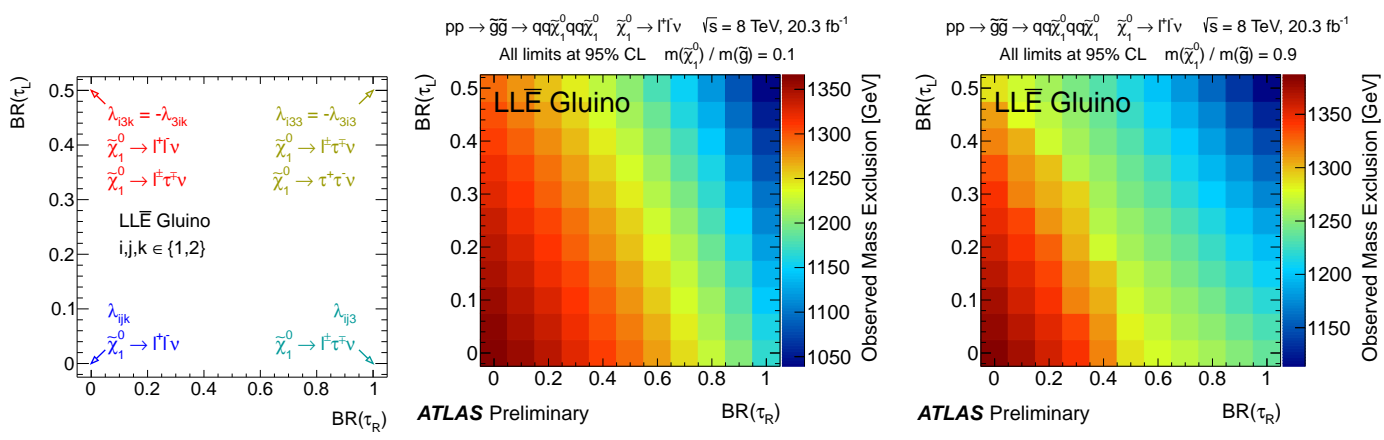

Figure 2: LLE gluino parameter space in $\lambda_{i j k}$ operators in which interpretations are done (left). Limit plots in the same parameter space for $\mathrm{m}\left(\widetilde{\chi}_{1}^{0}\right) / \mathrm{m}(\tilde{g})=0.1$ (center) and 0.9 (right)

\subsubsection{LQD gluino, squark}

Instead of decaying via the LLE operator, gluinos or squarks could also decay through the LQD operator, for example in the center diagram in Figure 1. This simplified model is treated similarly to the LLE case, except the reinterpretation is performed as a function of the branching ratios of the super-fields to tau leptons and b quarks, as shown in the top left plot of Figure 3. Limits for LQD gluino production for $\mathrm{m}\left(\widetilde{\chi}_{1}^{0}\right) / \mathrm{m}(\tilde{g})=0.1$ and 0.9 are shown in the top of Figure 3, and limits for LQD squark production for $\mathrm{m}\left(\widetilde{\chi}_{1}^{0}\right) / \mathrm{m}(\tilde{g})=0.5$ and 0.9 are shown in the bottom of Figure 3.

\subsubsection{Bilinear RPV}

Decays via the LH term in Equation 1.1 are denoted as bilinear RPV. In this model, the lightest supersymmetric particle (LSP) is a mass-degenerate neutralino/chargino pair. There are light top and bottom squarks, with all other supersymmetric particles pushed to kinematically inaccessible 


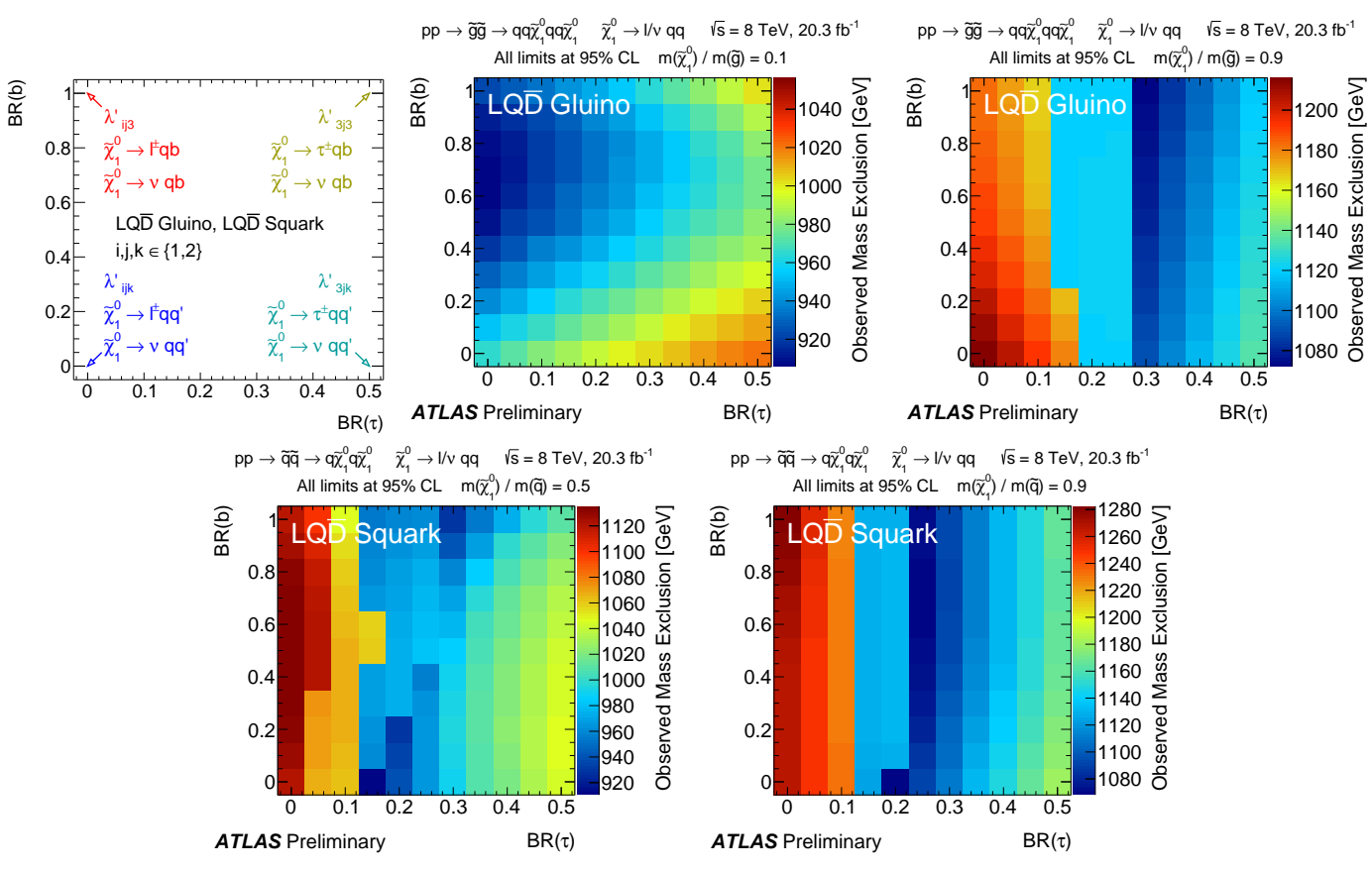

Figure 3: LQD gluino/squark parameter space in $\lambda_{i j k}$ operators in which interpretations are done (top left). Limit plots in the same parameter space for the LQD gluino models $\mathrm{m}\left(\widetilde{\chi}_{1}^{0}\right) / \mathrm{m}(\tilde{g})=0.1$ (top center) and 0.9 (top right). Limit plots in the same parameter space for the LQD squark models $\mathrm{m}\left(\tilde{\chi}_{1}^{0}\right) / \mathrm{m}(\tilde{g})=0.5$ (bottom left) and 0.9 (bottom right)

masses. A representative diagram of this model can be seen in Figure 4 left. Interpreted in the context of the pMSSM, this model is already constrained by neutrino experiments, by the mass of the Higgs boson, and by leptoquark reinterpretations with top squark LSP. Limits are set in a two dimensional plane as a function of the mass parameter for left-handed top and bottom squarks $\mathrm{m}_{\tilde{q}_{L, 3}}$, and the mass parameter which controls the neutralino/chargino mass $\mu$. The masses of the bottom and top squarks as a function of $\mathrm{m}_{\tilde{q}_{L, 3}}$ can be seen in the center plot of Figure 4 . 95\% confidence level limits can be seen as a function of $\mathrm{m}_{\tilde{q}_{L, 3}}$ and $\mu$ in the right-most plot of Figure 4 .
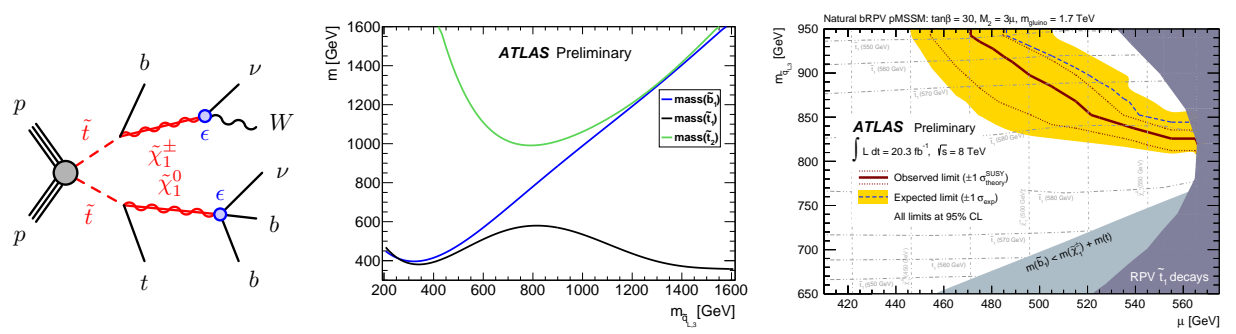

Figure 4: Representative bilinear RPV diagram (left). Masses of the bottom and top squarks as a function of $\mathrm{m}_{\tilde{q}_{L, 3}}$ (center). $95 \%$ confidence level limits as a function of $\mathrm{m}_{\tilde{q}_{L, 3}}$ and $\mu$. 


\subsection{UDD gluino}

Both ATLAS and CMS have recent results for searches using topologies which decay via the UDD operator, a representative diagram of which is shown as the right-most diagram in Figure 1. These topologies contain many jet in the final state, and searches are performed using both light and heavy flavor final states. The ATLAS search [5] uses two methods: a jet counting method, which requires at least 6 jets, with multiple b-jet categories; and a total jet mass method, which uses the scalar sum of the masses of the four leading large cone jets as a discriminant. The total jet mass of the data sample, along with backgrounds and a signal sample, can be seen on the left hand side of Figure 5. Limits are set on 6 jet and 10 jet signal models. The limits calculated using the total jet mass method for the 10 jet model, and as a function of gluino and neutralino masses, can be seen in the center plot of the same figure. The analysis is also interpreted as a function of the branching fraction of the gluino to individual quark flavors, the limits for which are shown on the right-hand side of Figure 5.
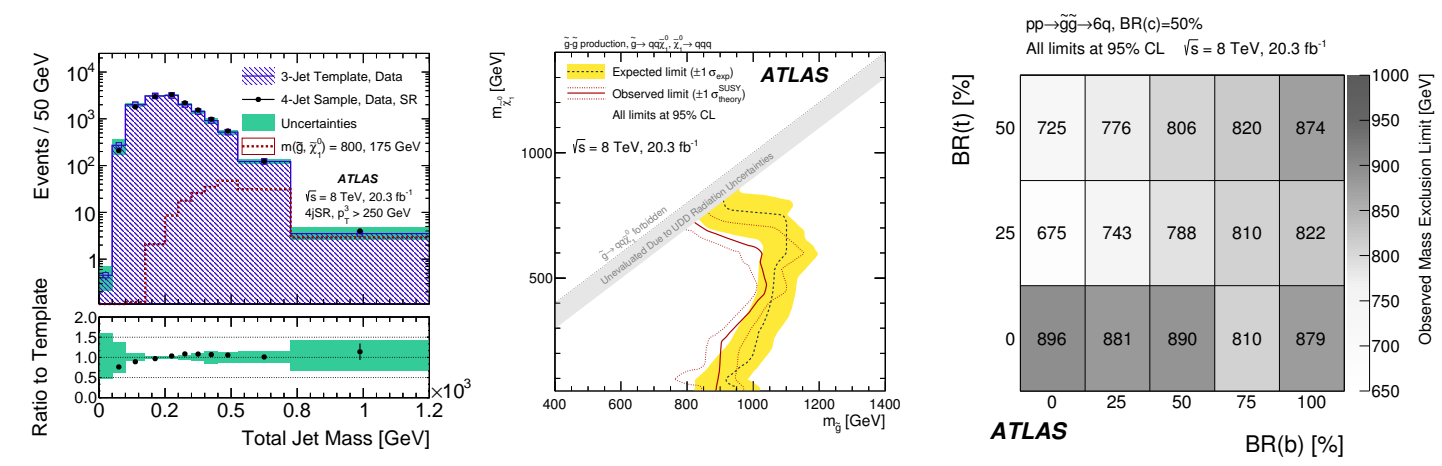

Figure 5: ATLAS UDD gluino search. Total jet mass of the data sample, along with backgrounds and a signal sample (left). Limits calculated using the total jet mass method for the 10 jet model as a function of gluino and neutralino masses (center). Limits set as a function of the branching fraction of the gluino to individual quark flavors (right).

A similar CMS search [6] considers only the 6 jet model, and performs the analysis as a 3 -jet resonance search, with 0 and $2 \mathrm{~b}$-tag signal regions. Limits for the 0 (2) b-tag signal regions as a function of 3-jet invariant mass can be seen on the left- (right-) hand side of Figure 6.

A second CMS search [7] directly targets heavy flavor gluino decay, performing a search which requires one lepton and searches for high-multiplicity jet and b-jet events. A signal diagram is shown in the left-most diagram of Figure 7. The center plots in Figure 7 show the data to background comparison of the number of b-tagged jets in the single electron (left) and muon (right) selections. Limits as a function of gluino mass are shown in the right-most plot of Figure 7.

\subsection{Top squarks and naturalness}

A search for so-called naturalness drives many SUSY models. These models tend to have light third-generation squarks (top and bottom squarks). 

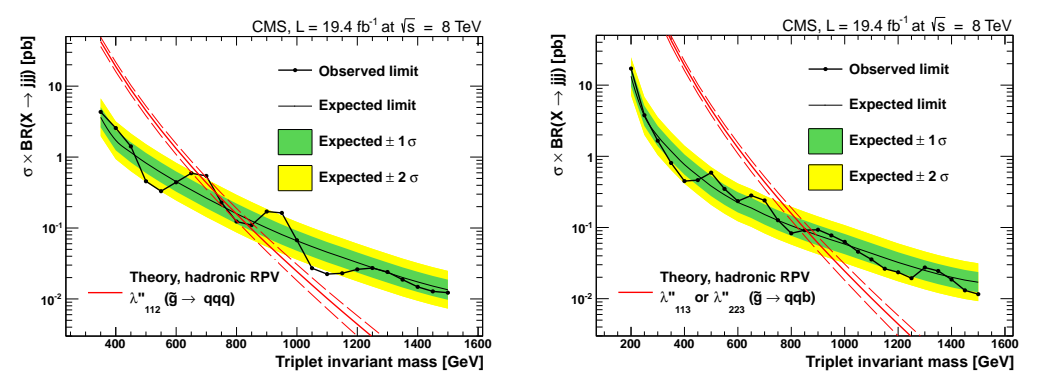

Figure 6: CMS UDD gluino search. Limits for the $0 \mathrm{~b}$-tag (left) and $2 \mathrm{~b}$-tag (right) signal regions as a function of 3-jet invariant mass.

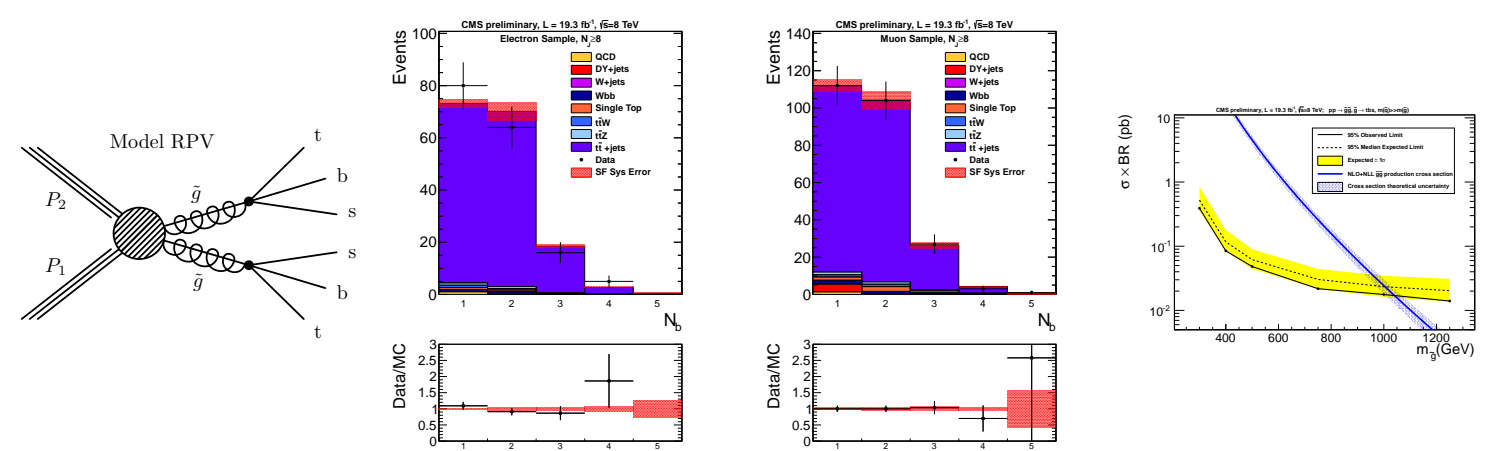

Figure 7: CMS UDD gluino heavy flavor search. Representative diagram (left). Data to background comparison of the number of b-tagged jets in the single electron (left center) and muon (right center) selections. Limits as a function of gluino mass (right).

\subsubsection{LQD leptoquark reinterpretation}

The $8 \mathrm{TeV}$ first and second generation pair-produced leptoquark result [8] is reinterpreted in the context of a Higgsino-mediated top squark decay [9]. The production and decay of this model is shown on the left-hand side of Figure 8. A Higgsino-mediated decay is considered because direct top squark decay is equivalent to leptoquark decay, and limits can be taken directly from the leptoquark searches.

\subsubsection{LQD top squark}

A CMS search [10] targets the chargino-mediated top squark decay shown in the top left diagram in Figure 9. This search requires two opposite-charged same-flavor electrons or muons, at least one b-tag, and split signal selections into 5, 6 and 7 jet categories. Each top squark mass signal point has an optimized $\mathrm{S}_{\mathrm{T}}$ cut, and the search is sensitive to the LQD operator $\lambda_{i j k}^{\prime}$ for all $\mathrm{i}, \mathrm{j}, \mathrm{k}<2$. The $\mathrm{N}_{\mathrm{jets}}$ distributions for the $e^{+} e^{-}$(left) and $\mu^{+} \mu^{-}$(right) selections are shown as the top plots in Figure 9, and the corresponding limit plots as a function of the top squark mass are shown in the bottom of the same figure. 

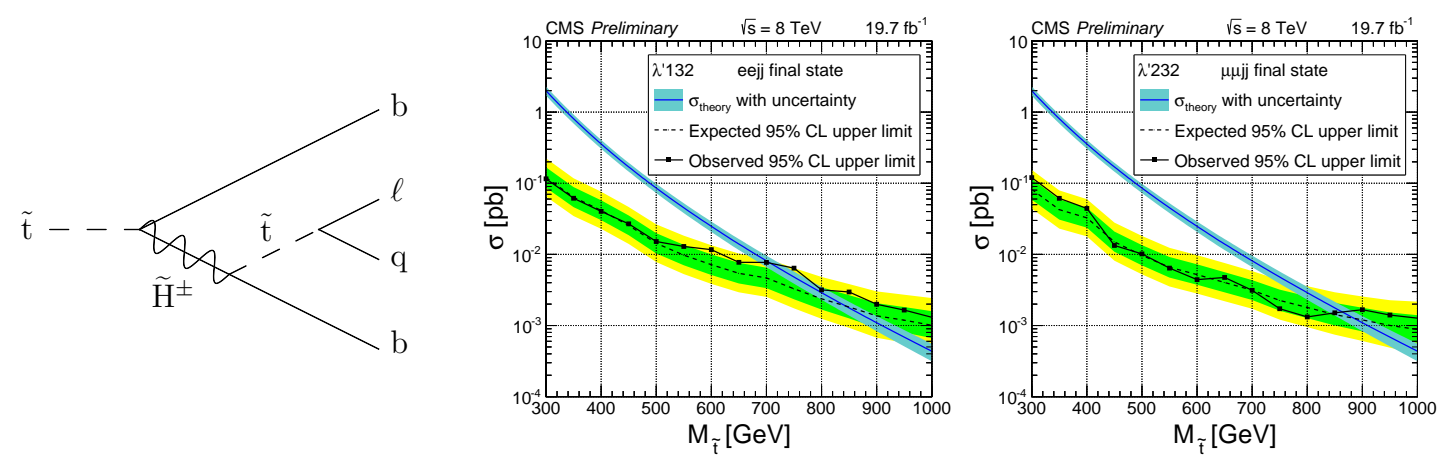

Figure 8: Diagram of the Higgsino-mediated top squark decay via the RPV $\lambda_{132}^{\prime}(\ell=e)$ or $\lambda_{232}^{\prime}(\ell=\mu)$ coupling (top). 95\% confidence level reinterpretation of leptoquark experimental limits on Higgsino-mediated stop production in the eejj (left) and $\mu \mu \mathrm{jj}$ (right) final states.
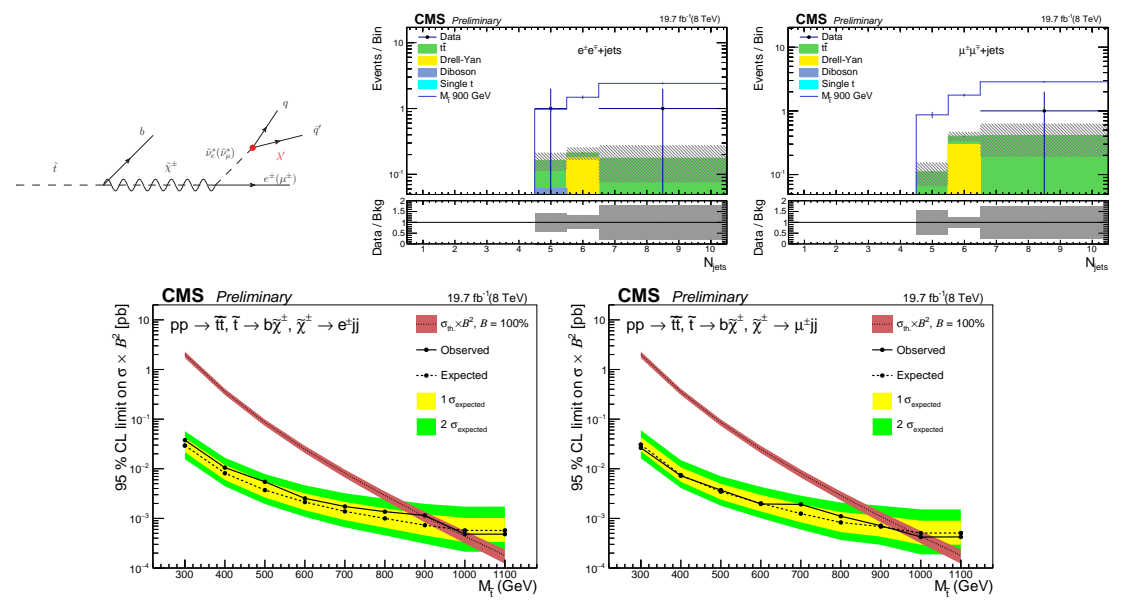

Figure 9: LQD chargino-mediated top squark decay (top left). $\mathrm{N}_{\text {jets }}$ distributions for the $e^{+} e^{-}$(top center) and $\mu^{+} \mu^{-}$(top right) selections. Limit plots as a function of the top squark mass for the $e^{+} e^{-}$(bottom left) and $\mu^{+} \mu^{-}$(bottom right) selections.

\subsubsection{UDD top squark}

An ATLAS search [11] targets low mass top squarks where the top squark decays via the $\lambda_{323}^{\prime \prime}$ operator to $\mathrm{b}$ and s quarks, as shown in the left-most diagram in Figure 10. The analysis looks for a boosted system with merged jets, using large cone jets and jet substructure techniques. The average jet mass is shown in the center plot of Figure 10, and limits as a function of top squark mass are shown on the right-most plot. This search provides a complement to high top squark mass searches.

\section{Summary}

ATLAS and CMS have a rich variety of analyses, with searches for all R-parity violating interactions. Many of the existing $8 \mathrm{TeV}$ limits are reaching or surpassing $1 \mathrm{TeV}$. Run 2 of the 

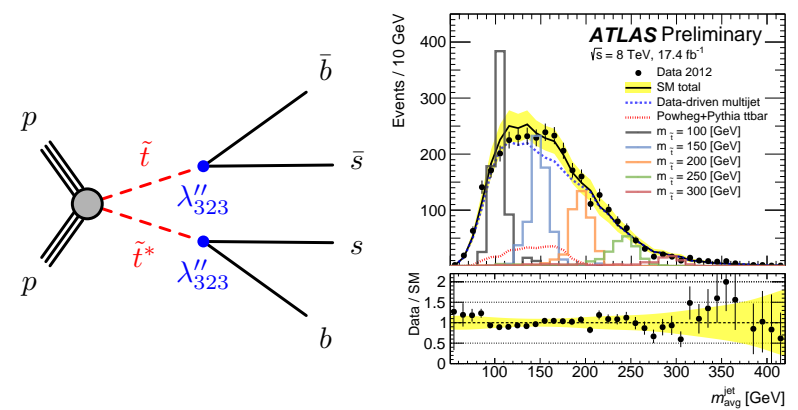

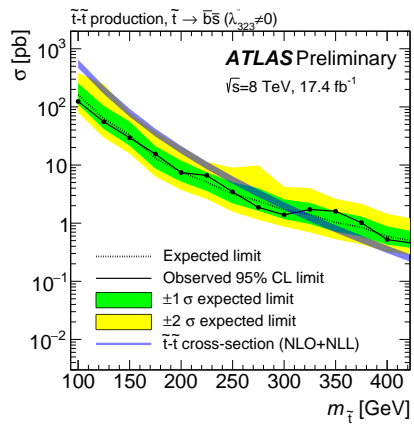

Figure 10: UDD top squark decay to $b$ and $s$ quarks (left). Average jet mass (center). Limits as a function of top squark mass (right).

LHC with a center of mass energy of $13 \mathrm{TeV}$ will extend the reach of all of these analyses, finding discoveries or tightening the allowable phase space for RPV SUSY, furthering our understanding of these models, of SUSY, and of the standard model.

\section{References}

[1] Martin, Stephen P., A Supersymmetry primer, Adv. Ser. Direct. High Energy Phys. 18, 1 (1998)

[2] ATLAS collaboration, A search for R-parity violating scalar top decays in all-hadronic final states with the ATLAS detector in $\sqrt{s}=8 \mathrm{TeV}$ pp collisions, ATLAS-CONF-2015-026

[3] ATLAS Collaboration, Constraints on promptly decaying supersymmetric particles with lepton-number-and R-parity-violating interactions using Run-1 ATLAS data, ATLAS-CONF-2015-018

[4] CMS Collaboration, Search for top squarks in R-parity violating supersymmetry with dileptons and jets, CMS-PAS-EXO-14-013

[5] ATLAS Collaboration, Search for massive supersymmetric particles decaying to many jets using the ATLAS detector in pp collisions at $\sqrt{s}=8$ TeV, Phys. Rev. D 91, 112016 (2015)

[6] CMS Collaboration, Searches for light-and heavy-flavour three-jet resonances in pp collisions at $\sqrt{s}$ $=8$ TeV, Phys. Lett. B 730 (2014) 193

[7] CMS Collaboration, A search for new physics in events with high jet and b-tagged jet multiplicities and one lepton, CMS-PAS-SUS-12-015

[8] CMS Collaboration, Search for pair production of first and second generation leptoquarks in proton-proton collisions at $\sqrt{s}=8 \mathrm{TeV}$, CMS-PAS-EXO-12-041, Submitted to Phys. Rev. D

[9] Jared A. Evans and Yevgeny Kats, LHC coverage of RPV MSSM with light stops, JHEP 04 (2013) 028

[10] CMS Collaboration, Search for top squarks in R-parity violating supersymmetry with dileptons and jets, CMS-PAS-EXO-14-013

[11] ATLAS Collaboration, A search for R-parity violating scalar top decays in all-hadronic final states with the ATLAS detector in $\sqrt{s}=8 \mathrm{TeV}$ pp collisions, ATLAS-CONF-2015-026 